\title{
Secondary criticality of water waves. Part 2. Unsteadiness and the Benjamin-Feir instability from the viewpoint of hydraulics
}

\author{
By T. J. BRIDGES AND N. M. DONALDSON \\ Department of Mathematics, University of Surrey, Guildford, Surrey, GU2 7XH, UK
}

(Received 5 November 2003 and in revised form 15 March 2006)

The theory for criticality presented in Part 1 is extended to the unsteady problem, and a new formulation of the Benjamin-Feir instability for Stokes waves in finite depth coupled to a mean flow, which takes the criticality matrix as an organizing centre, is presented. The generation of unsteady dark solitary waves at points of stability changes and their connection with the steady dark solitary waves of Part 1 are also discussed.

\section{Introduction}

A curiosity in the theory of water waves is the gap between concepts in hydraulics such as mass flux, total head, flow force and criticality and concepts associated with the time-dependent problem, such as the Benjamin-Feir (BF) instability. On the one hand, it is well known that it is mean flow that stabilizes the BF instability in shallow water, and clearly mean flow is analogous to a uniform flow. On the other hand, the connection between the hydraulic properties of uniform flows coupled to a Stokes waves and the BF instability has not been clearly established. The Whitham (1967) theory for BF instability in finite depth comes closest to linking these two concepts. The Whitham theory produces the correct result for BF instability, but it does not produce the parameter structure of uniform flows correctly. Specifically, it treats the total head as a pseudofrequency which is associated with time dependence, so in the limit as a steady flow is approached, the connection with uniform flows is lost (see $§ 7$ for elaboration).

Linking the properties of the time-dependent problem and the hydraulic properties of steady flows turns out to be possible by putting space and time on an equal footing whereby steady properties can be deformed in a natural way into the unsteady problem. This idea formed the basis of the new theory for criticality presented in Part 1 (Bridges \& Donaldson 2006), and is elaborated further here for the unsteady problem.

The main result of this paper is that a steady periodic Stokes wave in finite depth, coupled to uniform flow, is linearly unstable to long-wave perturbations if the determinant of the deformed criticality matrix, $\boldsymbol{N}(\Omega)$, has a root with non-zero imaginary part, where

$$
\boldsymbol{N}(\Omega)=\left[\begin{array}{lll}
\frac{\partial R}{\partial h_{0}} & \frac{\partial R}{\partial u_{0}} & \frac{\partial R}{\partial k} \\
\frac{\partial Q}{\partial h_{0}} & \frac{\partial Q}{\partial u_{0}} & \frac{\partial Q}{\partial k} \\
\frac{\partial B}{\partial h_{0}} & \frac{\partial B}{\partial u_{0}} & \frac{\partial B}{\partial k}
\end{array}\right]+\Omega\left[\begin{array}{ccc}
0 & 1 & \frac{\partial A}{\partial h_{0}} \\
1 & 0 & \frac{\partial A}{\partial u_{0}} \\
\frac{\partial A}{\partial h_{0}} & \frac{\partial A}{\partial u_{0}} & 2 \frac{\partial A}{\partial k}
\end{array}\right]+\Omega^{2}\left[\begin{array}{ccc}
0 & 0 & 0 \\
0 & 0 & 0 \\
0 & 0 & \frac{\partial A}{\partial \omega}
\end{array}\right] .
$$


The parameter $\Omega$ represents the stability exponent for the time-dependent problem. The determinant of $\mathbf{N}(\Omega)$ will be called the stability polynomial. It is a quartic polynomial in $\Omega$ when $A_{\omega} \neq 0$.

The most important feature of the above result is that the criticality matrix is at the centre of the stability polynomial. For example, it is clear that there is a zero root of the stability polynomial if and only if the basic state is critical.

The new function $A(Z)$ is related to the wave action, but is not precisely the wave action because it is evaluated on the steady problem. It is obtained by deformation of the variational principle for the steady waves, use of conservation of wave action, and then deforming back to the steady problem. All entries in the three matrices in $\boldsymbol{N}(\Omega)$ are evaluated on the steady wave. For example, to obtain $A_{\omega}$, the basic steady state is deformed to a modulated time-dependent state with frequency $\omega$, the wave action is evaluated on this deformed state, the derivative with respect to $\omega$ is taken, and then the limit $\omega \rightarrow 0$ is introduced, resulting in a function of the steady-state solution only. In fact, the wave action can be expressed in terms of the mass flux of the steady wave (cf. Appendix). The deformation and derivative provide information about the susceptibility of the basic steady-state wave to unsteadiness (see Bridges 2004 for a similar susceptibility argument arising in the stability analysis of bright solitary waves).

A sketch of how the theory proceeds is as follows. Consider a Hamiltonian PDE in multi-symplectic form

$$
\mathbf{M} Z_{t}+\mathbf{J} Z_{x}=\nabla S(Z), \quad Z \in \mathbb{H},
$$

where $\mathbb{H}$ represents the phase space. In (1.2), both $\boldsymbol{M}$ and $\boldsymbol{J}$ are skew-symmetric operators, and $S$ is a given smooth function. The symbol $S$ is used in anticipation that, for physical problems such as water waves and its model counterparts, coordinates are chosen so that $S$ is the flow force.

The steady part of (1.2)

$$
\boldsymbol{J}_{x}=\nabla S(Z), \quad Z \in \mathbb{H},
$$

is a classical Hamiltonian system in the $x$-direction. With coordinates chosen correctly (cf. Part 1), systems based on water waves have three invariants

$$
S_{x}=0, \quad R_{x}=0, \quad Q_{x}=0,
$$

where $S, Q$ and $R$ are precisely the physical quantities of flow force, mass flux and total head, and more importantly, in the Hamiltonian setting these bulk quantities are related to symmetry. Bringing the time-dependence back into the equation converts these invariants to fluxes of conservation laws,

$$
S_{x}+I_{t}=0, \quad Q_{x}+P_{t}=0, \quad R_{x}+F_{t}=0 .
$$

The first of these is the conservation of impulse which is discussed in detail in Benjamin (1984). The second is conservation of mass, and the third is the conservation form of Bernoulli's equation (the third conservation law is sometimes called conservation of energy, but conservation of energy for water waves takes a different form). Each of these conservation laws is associated with some symmetry via Noether's theorem. Conservation of impulse is associated with translation invariance in the $x$-direction of the governing equations. Conservation of mass is associated with translation of the velocity potential - that is, an arbitrary function of time can be added to the velocity potential. Heretofore, the association of the third conservation law with a symmetry has gone unnoticed. It is a hidden symmetry which becomes apparent 
when a potential is introduced for the free-surface elevation. This new symmetry was introduced in Part 1 for steady waves, and is extended to the unsteady problem in $\S 2$.

Let $A(Z)$ represent the wave action. Then, adding in conservation of wave action, $\partial_{t} A+\partial_{x} B=0$, to (1.3) leads to a set of 8 functionals: four spatial (the fluxes), which are central to the steady problem, and four temporal functionals, which come into play in the analysis of the unsteady equations.

To motivate the importance of the embedding of the steady wave in the unsteady problem, the linear stability problem is formulated for the steady waves of Part 1 , without embedding. The required deformation will then arise naturally, and the theory for this is given in $\S \S 4$ and 5. The linear stability analysis for long-wave instabilities is then completed in $\S 6$. The similarities and differences with the instability theory of Whitham are then considered in $\S 7$. In $\S 8$, it is shown that the stability polynomial obtained from (1.1) reproduces the classical stabilization of the BF instability due to mean flow which occurs in the weakly nonlinear limit. Some comments on bandwidth of unstable wavenumbers are given in $\S 9$. The final section gives an overview and summarizes the results of Parts 1 and 2.

\section{A Boussinesq model for modelling unsteady criticality}

A Boussinesq model will be used to illustrate the theory of unsteady criticality. Since this paper will restrict attention to stability of periodic waves, the Boussinesq model with third-order dispersion is sufficient. An exemplar in this class which is sufficient for the present purposes is,

$$
\left.\begin{array}{l}
h_{t}+u h_{x}+h u_{x}+\frac{1}{3} u_{x x x}=0, \\
u_{t}+u u_{x}+g h_{x}-\tau h_{x x x}=0,
\end{array}\right\}
$$

where, to match the dispersion relation for water waves we require $\tau$ to be proportional to the coefficient of surface tension (equivalently, proportional to the Bond number) (cf. Dingemans 1997, pp. 507, 515). (The presence of surface tension in the model (2.1) is not important for the later discussion of unsteady criticality or stability of water waves, as the interest is predominantly in gravity waves. However, surface tension is straightforward to include, and simplifies the structure of the equations.) For some parameter values these equations are ill-posed and can be replaced by other members of the Boussinesq equivalence class. However, since the system (2.1) is used in this paper for illustration only, and the applications later will be to the full water-wave problem, this ill-posedness is not important.

Extending the idea of Part 1, a potential for both the velocity and the surface elevation is introduced,

$$
u(x, t)=\frac{\partial}{\partial x} \phi(x, t), \quad h(x, t)=\frac{\partial}{\partial x} \gamma(x, t) .
$$

Now, by introducing new variables $\eta=\tau h_{x}, v=-u_{x} / 3, q$ and $r$ (defined below), it is easily verified that the Boussinesq model (2.1) is equivalent to the system of first-order PDEs

$$
\left.\begin{array}{rlrl}
-r_{x} & =0, & & \gamma_{x}=h, \\
-h_{t}-q_{x} & =0, & & \phi_{x}=u, \\
\phi_{t}-\eta_{x} & =r-g h-\frac{1}{2} u^{2}, & & h_{x}=\eta / \tau, \\
-v_{x} & =q-h u, & & u_{x}=-3 v,
\end{array}\right\}
$$


and this system can be written in standard form for a multi-symplectic PDE

$$
\boldsymbol{M} Z_{t}+J_{Z_{x}}=\nabla S(Z), \quad Z \in \mathbb{R}^{8},
$$

where $Z=(\gamma, \phi, h, u, r, q, \eta, v) \in \mathbb{R}^{8}$,

$$
\boldsymbol{M}=\left[\begin{array}{cccccccc}
0 & 0 & 0 & 0 & 0 & 0 & 0 & 0 \\
0 & 0 & -1 & 0 & 0 & 0 & 0 & 0 \\
0 & 1 & 0 & 0 & 0 & 0 & 0 & 0 \\
0 & 0 & 0 & 0 & 0 & 0 & 0 & 0 \\
0 & 0 & 0 & 0 & 0 & 0 & 0 & 0 \\
0 & 0 & 0 & 0 & 0 & 0 & 0 & 0 \\
0 & 0 & 0 & 0 & 0 & 0 & 0 & 0 \\
0 & 0 & 0 & 0 & 0 & 0 & 0 & 0
\end{array}\right], \quad \boldsymbol{J}=\left[\begin{array}{cccccccc}
0 & 0 & 0 & 0 & -1 & 0 & 0 & 0 \\
0 & 0 & 0 & 0 & 0 & -1 & 0 & 0 \\
0 & 0 & 0 & 0 & 0 & 0 & -1 & 0 \\
0 & 0 & 0 & 0 & 0 & 0 & 0 & -1 \\
1 & 0 & 0 & 0 & 0 & 0 & 0 & 0 \\
0 & 1 & 0 & 0 & 0 & 0 & 0 & 0 \\
0 & 0 & 1 & 0 & 0 & 0 & 0 & 0 \\
0 & 0 & 0 & 1 & 0 & 0 & 0 & 0
\end{array}\right],
$$

and

$$
S(Z)=r h+q u-\frac{1}{2} h u^{2}-\frac{1}{2} g h^{2}+\frac{1}{2 \tau} \eta^{2}-\frac{3}{2} v^{2} .
$$

The steady conservation equations of $\S 2$ of Part 1 extend to unsteady conservation laws. Let $\mathfrak{g}_{1}=\boldsymbol{e}_{1}$ and $\mathfrak{g}_{2}=\boldsymbol{e}_{2}$ where $\boldsymbol{e}_{j}$ be the standard $j$ th unit vector in $\mathbb{R}^{8}$. Then, the fact that $S(Z)$ is independent of $\gamma$ and $\phi$ can be represented by the statement

$$
S(\mathrm{G}(\boldsymbol{\theta}) Z)=S(Z), \quad \forall \boldsymbol{\theta} \in \mathbb{R}^{2} .
$$

where

$$
\mathrm{G}(\boldsymbol{\theta}) Z=Z+\theta_{1} \mathfrak{g}_{1}+\theta_{2} \mathfrak{g}_{2}, \quad \forall \boldsymbol{\theta}=\left(\theta_{1}, \theta_{2}\right) \in \mathbb{R}^{2} .
$$

The above two-parameter group of symmetries can be related to the conservation of mass and Bernoulli's equation as follows. Let $\langle\cdot, \cdot\rangle$ be a standard inner product on $\mathbb{R}^{8}$, and define

$$
R(Z)=\left\langle\mathfrak{J}_{\mathfrak{g}_{1}}, Z\right\rangle=\left\langle\boldsymbol{e}_{5}, Z\right\rangle=r \quad \text { and } \quad Q(Z)=\left\langle\mathrm{Jg}_{2}, Z\right\rangle=\left\langle\boldsymbol{e}_{6}, Z\right\rangle=q .
$$

Then

$$
\frac{\partial}{\partial x} R(Z)=\left\langle\mathbf{J g}_{1}, Z_{x}\right\rangle=-\left\langle\mathfrak{g}_{1}, \mathbf{J} Z_{x}\right\rangle=-\left\langle\mathfrak{g}_{1}, \nabla S(Z)-\boldsymbol{M} Z_{t}\right\rangle
$$

and so

$$
\frac{\partial}{\partial t}\left\langle\boldsymbol{M g}_{1}, Z\right\rangle+\frac{\partial}{\partial x} R(Z)=-\left.\frac{\partial}{\partial \theta_{1}} S(\mathrm{G}(\boldsymbol{\theta}) Z)\right|_{\theta=0}=0 ;
$$

resulting in Bernoulli's equation in conservation form, a conservation law for total head,

$$
F_{t}+R_{x}=0 \quad \text { with } \quad F=\left\langle\boldsymbol{M} \mathfrak{g}_{1}, Z\right\rangle .
$$

However, because of the special form of $\boldsymbol{M}$, the vector $\mathfrak{g}_{1}$ is in the kernel of $\boldsymbol{M}$ and so $F=0$ and conservation of total head has a flux only: $R_{x}=0$, even in the timedependent problem. A geometric formulation of conservation of mass can be derived similarly leading to

$$
P_{t}+Q_{x}=0 \quad \text { with } \quad P(Z)=\left\langle\boldsymbol{M g}_{2}, Z\right\rangle=h .
$$

The geometric formulation of the conservation of wave action is extended to the unsteady problem as follows. Let $Z(x, t, s)$ be a closed curve (an ensemble) of 
solutions of (2.4), parameterized by $s$; that is, $Z(x, t, s+2 \pi)=Z(x, t, s)$ and $Z(x, t, s)$ satisfies (2.4) for each $s$ with $0 \leqslant s<2 \pi$. Then $\partial_{t} A(Z)+\partial_{x} B(Z)=0$ with

$$
A(Z)=\frac{1}{2} \oint\left\langle\boldsymbol{M} Z_{s}, Z\right\rangle \mathrm{d} s, \quad B(Z)=\frac{1}{2} \oint\left\langle\mathbf{J} Z_{s}, Z\right\rangle \mathrm{d} s, \quad \oint \cdot \mathrm{d} s:=\frac{1}{2 \pi} \int_{0}^{2 \pi} \cdot \mathrm{d} s .
$$

\section{Long-wave stability of periodic waves on a uniform flow}

In this section, the linear stability problem for the three-parameter family of steady waves introduced in Part 1 is formulated.

For simplicity of presentation, the theory will be developed in detail for the Boussinesq system in multi-symplectic form introduced in $\S 2$, and then results of the theory for water waves will be discussed.

The theory is in fact based only on the multi-symplectic form of the governing equations with the symmetries as discussed in $\S 2$,

$$
M Z_{t}+\mathbf{J} Z_{x}=\nabla S(Z), \quad Z \in \mathbb{H},
$$

where $\boldsymbol{M}$ and $\boldsymbol{J}$ are skew symmetric, and $\mathbb{H}$ is the phase space, which is an inner product space, and for simplicity will be assumed finite-dimensional here.

Consider a perturbation of the basic state of $\S 5$ of Part 1 , that is, let

$$
Z(x, t)=G(\boldsymbol{\theta}(x))[\widehat{Z}+\widehat{U}]:=\widehat{Z}\left(\theta_{3}\right)+\widehat{U}\left(\theta_{3}, x, t\right)+\theta_{1}(x) \mathfrak{g}_{1}+\theta_{2}(x) \mathfrak{g}_{2} .
$$

Substituting this expression into (3.1) and linearizing about the basic state $\widehat{Z}$ leads to the linear constant coefficient PDE

$$
\mathbf{M} \widehat{U}_{t}+\boldsymbol{J} \widehat{U}_{x}=\mathbf{L}(\boldsymbol{p}) \widehat{U}
$$

where

$$
\boldsymbol{L}(\boldsymbol{p})=\mathrm{D}^{2} S(\widehat{Z})-k \mathrm{D}^{2} B(\widehat{Z}),
$$

taking into account that $R$ and $Q$ are linear functions of $\widehat{Z}$.

From equation (B3) in Appendix B of Part 1, it is immediate that $\operatorname{span}\left\{\mathfrak{g}_{1}, \mathfrak{g}_{2}, \mathfrak{g}_{3}\right\} \subseteq$ Kernel $(\boldsymbol{L}(\boldsymbol{p}))$ and we will take it as a hypotheses that the Kernel is not larger, i.e.

$$
\operatorname{span}\left\{\mathfrak{g}_{1}, \mathfrak{g}_{2}, \mathfrak{g}_{3}\right\}=\operatorname{Kernel}(\boldsymbol{L}(\boldsymbol{p})) .
$$

The operator $\boldsymbol{L}(\boldsymbol{p})$ is a differential operator in $\theta_{3}$, and the coefficients (for example $\left.\mathrm{D}^{2} S\left(\widehat{Z}\left(\theta_{3}\right)\right)\right)$ depend periodically on $\theta_{3}$. Therefore in analysing the linear stability problem, we take a class of periodic perturbations of Floquet type in the $x$-direction. Let

$$
\widehat{U}\left(\theta_{3}, x, t\right)=U_{1}\left(\theta_{3}, t\right) \cos \alpha x+U_{2}\left(\theta_{3}, t\right) \sin \alpha x,
$$

for some $\alpha \in \mathbb{R}$, and where $U_{j}\left(\theta_{3}, t\right)$ are $2 \pi$-periodic functions of $\theta_{3}$.

The representation (3.3) is much weaker than a proper 'Floquet decomposition'. The Floquet decomposition states that every solution is of the form of an exponential multiplied by a function of the same period as the basic state. Here, the aim is to show that there exists at least one solution of the form (3.3), and since the periodic direction is spatial, the solution should be bounded: the Floquet exponent should be purely imaginary and hence $\alpha \in \mathbb{R}$, leading to the sin-cos representation. 
Now, look for temporally exponential solutions, $\left(U_{1}\left(\theta_{3}, t\right), U_{2}\left(\theta_{3}, t\right)\right)=\left(W_{1}\left(\theta_{3}\right)\right.$, $\left.W_{2}\left(\theta_{3}\right)\right) \mathrm{e}^{\lambda t}$; then we have the following spectral problem

$$
\left[\begin{array}{cc}
\boldsymbol{L}(\boldsymbol{p}) & -\alpha \boldsymbol{J} \\
\alpha \boldsymbol{J} & \boldsymbol{L}(\boldsymbol{p})
\end{array}\right]\left(\begin{array}{l}
W_{1} \\
W_{2}
\end{array}\right)=\lambda\left[\begin{array}{cc}
\boldsymbol{M} & \mathbf{0} \\
\mathbf{0} & \boldsymbol{M}
\end{array}\right]\left(\begin{array}{l}
W_{1} \\
W_{2}
\end{array}\right), \quad W=\left(\begin{array}{l}
W_{1} \\
W_{2}
\end{array}\right) \in \mathbb{H}_{\mathbb{C}} \times \mathbb{H}_{\mathbb{C}},
$$

where $\mathbb{H}_{\mathbb{C}}$ is the complex version of $\mathbb{H}$. The analysis of the spectral problem (3.4) is simplified by noting that the block system is easily diagonalized. Let

$$
\boldsymbol{T}=\frac{1}{\sqrt{2}}\left(\begin{array}{cc}
-\mathrm{i} \boldsymbol{l} & \mathrm{i} \boldsymbol{l} \\
\boldsymbol{I} & \boldsymbol{I}
\end{array}\right) ; \quad \text { then } \quad \boldsymbol{T}^{*}\left[\begin{array}{cc}
\mathbf{0} & -\boldsymbol{I} \\
\boldsymbol{I} & \mathbf{0}
\end{array}\right] \boldsymbol{T}=\operatorname{diag}(-\mathrm{i} \boldsymbol{l}, \mathrm{i} \boldsymbol{l})
$$

and so, by introducing the new variable $W=\boldsymbol{T} \zeta$ with $\zeta=\left(\boldsymbol{\zeta}_{-}, \zeta_{+}\right)$, and multiplying (3.4) by $\boldsymbol{T}^{*}$, it is block diagonalized to

$$
\left[\begin{array}{cc}
\boldsymbol{L}(\boldsymbol{p})-\lambda \boldsymbol{M}-\mathrm{i} \alpha \boldsymbol{J} & \mathbf{0} \\
\mathbf{0} & \boldsymbol{L}(\boldsymbol{p})-\lambda \boldsymbol{M}+\mathrm{i} \alpha \boldsymbol{J}
\end{array}\right]\left(\begin{array}{l}
\boldsymbol{\zeta}_{-} \\
\zeta_{+}
\end{array}\right)=\left(\begin{array}{l}
0 \\
0
\end{array}\right) .
$$

Hence the spectral problem decouples into two lower-order spectral problems which can be solved independently. Therefore, the basic spectral problem is

$$
L(p) \zeta_{-}=\lambda M \zeta_{-}+\mathrm{i} \alpha J \zeta_{-}
$$

The linear stability problem, for a basic wave in the family of steady waves interacting with a uniform flow of Part 1, reduces to studying the spectral problem (3.6). If there is a solution $\zeta_{-}$satisfying the eigenvalue problem (3.6), for some $\alpha \in \mathbb{R}$, with $\operatorname{Re}(\lambda)>0$ then we say the basic state is linearly unstable or spectrally unstable.

The spectral problem (3.6) has a very nice structure. The operator $\boldsymbol{L}(\boldsymbol{p})$ is symmetric and it is the second variation of the constrained variational principle for the basic state, $\boldsymbol{M}$ and $\boldsymbol{J}$ are skew symmetric and so i $\boldsymbol{J}$ is Hermitian.

A complete analysis of this spectral problem is beyond the scope of this paper, but by restricting to long-wave instabilities - that is, $\alpha$ small - fairly complete results can be obtained.

To determine the long-wave instabilities, the strategy will be to expand $\zeta_{-}$in a Taylor series in $\lambda$ and $\alpha$, and then use the solvability condition for (3.6) to determine the leading-order terms in the relationship between $\lambda$ and $\alpha$. The leading-order Taylor expansion of $\zeta_{-}$is

$$
\begin{aligned}
\zeta_{-}=c_{1}\left(\mathfrak{g}_{1}+\lambda V_{11}+\mathrm{i} \alpha V_{12}+\cdots\right)+c_{2}\left(\mathfrak{g}_{2}\right. & \left.+\lambda V_{21}+\mathrm{i} \alpha V_{22}+\cdots\right) \\
& +c_{3}\left(\mathfrak{g}_{3}+\lambda V_{31}+\mathrm{i} \alpha V_{32}+\cdots\right),
\end{aligned}
$$

where the higher-order terms are higher order in $|\lambda|$ and $|\alpha|$. Substitution of this expression into (3.6), and using the fact that $\mathfrak{g}_{j}, j=1,2,3$ are in the kernel of $\boldsymbol{L}(\boldsymbol{p})$ shows that the first-order (in $\lambda$ and $\alpha$ ) terms satisfy

$$
\boldsymbol{L}(\boldsymbol{p}) V_{j 1}=\boldsymbol{M} \mathfrak{g}_{j}, \quad \boldsymbol{L}(\boldsymbol{p}) V_{j 2}=\mathbf{J g}_{j}, \quad j=1,2,3 .
$$

It follows from equations (B3) and (B4) in Appendix B of Part 1 that there are explicit expressions for $V_{j 2}$,

$$
V_{12}=\frac{\partial \widehat{Z}}{\partial h_{0}}+\operatorname{Ker}(\boldsymbol{L}(\boldsymbol{p})), \quad V_{22}=\frac{\partial \widehat{Z}}{\partial u_{0}}+\operatorname{Ker}(\boldsymbol{L}(\boldsymbol{p})), \quad V_{32}=\frac{\partial \widehat{Z}}{\partial k}+\operatorname{Ker}(\boldsymbol{L}(\boldsymbol{p})) .
$$


The term $+\operatorname{Ker}(\boldsymbol{L}(\boldsymbol{p}))$ is an indication that an arbitrary element in the kernel of $\boldsymbol{L}(\boldsymbol{p})$ can be added to the solution. However, these elements will drop out in the later calculation and so are not made explicit.

It remains to solve for $V_{j 1}$ with $j=1,2,3$. When $j=1$ the equation trivializes since $\mathfrak{g}_{1}$ is in the kernel of $\boldsymbol{M}$. Hence we need to solve for $V_{21}$ and $V_{31}$ only. The equations $\boldsymbol{L}(\boldsymbol{p}) V_{j 1}=\boldsymbol{M g}_{j}$ for $j=2,3$ are certainly solvable. They satisfy the solvability conditions,

$$
\oint\left\langle\mathfrak{g}_{i}, \boldsymbol{M g}_{j}\right\rangle \mathrm{d} \theta_{3}=0, \quad i, j=2,3,
$$

since $\boldsymbol{M g}_{2}=\nabla P$ and $\boldsymbol{M g}_{3}=\nabla A$ and the functionals $P$ and $A$ are invariant.

How to solve for $V_{21}$ and $V_{31}$ without using brute force? It is precisely this impasse which suggests that we need to deform the basic state into the unsteady problem. One interpretation of this deformation is that the stability problem requires information about how sensitive the basic state is to temporal modulation.

\section{Towards criticality for unsteady waves}

The key step in going from criticality of uniform flows to criticality of Stokes waves in Part 1 was the characterization of the basic state as a relative equilibrium. Criticality was then associated with a degenerate relative equilibrium.

Relative equilibria can be extended to include time dependence, by embedding the relative equilibrium in a multi-symplectic relative equilibrium (cf. Bridges 1997). To keep technicalities to a minimum, the analysis is carried out for the multi-symplectic form of the Boussinesq equation.

The starting point is (3.1). The three-parameter family of waves of $\S 5$ of Part 1 is generalized to

$$
Z(x, t)=\mathrm{G}(\boldsymbol{\theta}(x, t)) \widehat{Z}:=\widehat{Z}\left(\theta_{3}\right)+\theta_{1}(x, t) \mathfrak{g}_{1}+\theta_{2}(x, t) \mathfrak{g}_{2},
$$

with $\theta_{1}, \theta_{2}$ and $\theta_{3}$ affine functions of both $x$ and $t$,

$$
\theta_{1}(x, t)=h_{0} x+b t+\theta_{1}^{0}, \quad \theta_{2}(x, t)=u_{0} x+a t+\theta_{2}^{0}, \quad \theta_{3}(x, t)=k x+\omega t+\theta_{3}^{0} .
$$

The parameters $\theta_{1}^{0}, \theta_{2}^{0}$ and $\theta_{3}^{0}$ are arbitrary phase constants, and $(a, b, \omega)$ are the generalized speed along the group associated with the time direction.

For the Boussinesq model and water waves, it is found a posteriori that $b=0$. The parameter $b$ is zero because the conservation law for total head has only one component (a flux only). Therefore set $b=0$ from the outset.

The space-time properties are now coupled. To determine $(\widehat{Z}, \boldsymbol{p}, \omega, a)$, substitute (4.1) in (3.1) to find

$$
\nabla S(\widehat{Z})=h_{0} \nabla R(\widehat{Z})+u_{0} \nabla Q(\widehat{Z})+k \nabla B(\widehat{Z})+a \boldsymbol{M} \mathfrak{g}_{2}+\omega \boldsymbol{M} \frac{\partial \widehat{Z}}{\partial \theta_{3}} .
$$

But,

$$
\begin{aligned}
\boldsymbol{M g}_{2} & =\nabla P(\widehat{Z}) \quad \text { with } \quad P(\widehat{Z})=\oint\left\langle\boldsymbol{M g}_{2}, \widehat{Z}\right\rangle \mathrm{d} \theta_{3}, \\
\boldsymbol{M} \frac{\partial \widehat{Z}}{\partial \theta_{3}} & =\nabla A(\widehat{Z}) \quad \text { with } \quad A(\widehat{Z})=\oint \frac{1}{2}\left\langle\boldsymbol{M} \frac{\partial \widehat{Z}}{\partial \theta_{3}}, \widehat{Z}\right\rangle \mathrm{d} \theta_{3} .
\end{aligned}
$$

Hence the program is the same as before, we merely have additional Lagrange multipliers. The function $\widehat{Z}\left(\theta_{3}\right)$ is obtained as a critical point of $S$ restricted to level 
sets of the 5 functionals

$$
R(\widehat{Z})=\mathscr{R}, \quad Q(\widehat{Z})=\mathscr{Q}, \quad B(\widehat{Z})=\mathscr{B}, \quad A(\widehat{Z})=\mathscr{A}, \quad P(\widehat{Z})=\mathscr{P},
$$

with $\mathscr{R}, \mathscr{Q}, \mathscr{B}, \mathscr{A}$ and $\mathscr{P}$ specified. This constrained variational principle is nondegenerate when

$$
\left|\frac{\partial(R, Q, B, A, P)}{\partial(h, u, k, \omega, a)}\right| \neq 0,
$$

suppressing again the 0 -subscript in the derivatives. For both the Boussinesq model and the full water-wave problem, the matrix in (4.3) can be simplified. For example, in the Boussinesq model, the functional $P(\widehat{Z})$ takes the form

$$
P(\widehat{Z})=\oint\left\langle\boldsymbol{M} \mathfrak{g}_{2}, \widehat{Z}\right\rangle \mathrm{d} \theta_{3}=\oint \widehat{h}\left(\theta_{3}\right) \mathrm{d} \theta_{3} .
$$

But $\widehat{h}\left(\theta_{3}\right)$ is periodic, and the mean value of $\widehat{h}\left(\theta_{3}\right)$ is $h_{0}$. Hence $P(\widehat{Z})=h_{0}$. (A similar result holds for water waves.) Therefore the non-degeneracy condition in (4.3) simplifies to

$$
0 \neq \operatorname{det}\left[\begin{array}{ccccc}
R_{h} & R_{u} & R_{k} & R_{\omega} & 1 \\
Q_{h} & Q_{u} & Q_{k} & Q_{\omega} & 0 \\
B_{h} & B_{u} & B_{k} & B_{\omega} & 0 \\
A_{h} & A_{u} & A_{k} & A_{\omega} & 0 \\
1 & 0 & 0 & 0 & 0
\end{array}\right]=-\left|\frac{\partial(Q, B, A)}{\partial\left(u_{0}, k, \omega\right)}\right|
$$

using standard results for the determinant of a bordered matrix (e.g. Magnus \& Neudecker 1988, p. 43), where subscripts are used to denote partial derivatives.

The class of solutions (4.1) is quasi-periodic in time when viewed from a fixed frame of reference, since there are two independent frequencies: $\omega$ and $k u_{0}$, and the drift along the potentials is linear in time. In the limit as $a \rightarrow 0$ and $\omega \rightarrow 0$, this state reduces precisely to the spatially periodic steady wave on a uniform flow. According to the above derivation, the time-dependent state is degenerate - and hence critical when the determinant condition (4.4) fails to be satisfied.

To find whether the degeneracy in this case is significant, we can evaluate the non-degeneracy condition (4.4) in the limit as $\left|A_{1}\right| \rightarrow 0$.

Extending the weakly nonlinear analysis of $\S 5$ of Part 1 to include the two new functionals $P(Z)$ and $A(Z)$, leads to a weakly nonlinear equation with $D(\boldsymbol{p})$ of $\S 5$ of Part 1 replaced by $D(\boldsymbol{p}, \omega, a)$, using the same symbol, $D$, for the extended dispersion relation. Noting that to leading order $A=-D_{\omega}\left|A_{1}\right|^{2}+\cdots$, the determinant in (4.4) reduces to

$$
\left|\frac{\partial(Q, B, A)}{\partial\left(u_{0}, k, \omega\right)}\right|=\frac{h_{0}}{\varrho}\left|A_{1}\right|^{2} \operatorname{det}\left[\begin{array}{ccc}
D_{\omega \omega} & D_{\omega k} & D_{\omega} \\
D_{k \omega} & D_{k k} & D_{k} \\
D_{\omega} & D_{k} & 0
\end{array}\right]+O\left(\left|A_{1}\right|^{4}\right) .
$$

Hence, for non-degeneracy we require $h_{0} \neq 0, \varrho \neq 0$ (which is already required for the weakly nonlinear existence of steady waves), and a third condition based on the derivatives of $D$. The determinant in the third term can be related to the curvature of the dispersion curve, $\omega^{\prime \prime}(k)$.

Differentiating $D(\omega, k)=0$ (suppressing the other parameters in the dispersion relation for this discussion, as only $\omega$ and $k$ enter), and assuming $D_{\omega} \neq 0$, then 
$D_{\omega} \omega^{\prime}(k)+D_{k}=0$ and

$$
D_{\omega} \omega^{\prime \prime}(k)+D_{\omega \omega} \omega^{\prime}(k)^{2}+2 D_{\omega k} \omega^{\prime}(k)+D_{k k}=0 .
$$

Hence, substituting for $\omega^{\prime}(k)$,

$$
\omega^{\prime \prime}(k)=\frac{1}{D_{\omega}^{3}} \widehat{\delta}, \quad \widehat{\delta}:=\operatorname{det}\left[\begin{array}{ccc}
D_{\omega \omega} & D_{\omega k} & D_{\omega} \\
D_{k \omega} & D_{k k} & D_{k} \\
D_{\omega} & D_{k} & 0
\end{array}\right] .
$$

The non-degeneracy of the determinant in (4.5) is therefore equivalent to $\omega^{\prime \prime}(k) \neq 0$ (when $D_{\omega} \neq 0$ ). Therefore if we consider the frequency of the form $\omega(k)=\omega_{0}(k)+$ $\omega_{2}(k)\left|A_{1}\right|^{2}+\cdots$, then $\operatorname{sign}\left(\omega_{2}\right)=-\operatorname{sign}(\varrho)$, and so

$$
\operatorname{sign}\left(\left|\frac{\partial(Q, B, A)}{\partial\left(u_{0}, k, \omega\right)}\right|\right)=-\operatorname{sign}\left(\omega_{2} \omega_{0}^{\prime \prime}(k)\right)
$$

for $\left|A_{1}\right|$ sufficiently small. Now $\omega_{2} \omega_{0}^{\prime \prime}(k)<0$ is the Whitham (1974) condition for instability of weakly nonlinear dispersive waves. Hence, it might appear that when the determinant in (4.5) is positive, it signals an instability, and when the determinant changes sign, it signals a change of stability, suggesting that criticality of the unsteady wave implies stability change. However, although the determinant (4.5) does enter the linear stability problem, the stability analysis is more subtle and requires more detailed study. Briefly, the condition (4.7) fails to take account the effect of mean flow on stability.

\section{Deforming the basic steady flow into an unsteady flow}

In this section, it is shown how the deformation introduced in $\S 4$ can be used to determine the unknown functions $V_{j 1}$ in the linear stability problem.

There is now enough information to determine the functions $V_{21}$ and $V_{31},(3.8)$. Differentiate (4.2) with respect to $\omega$ and $a$, and use the fact that $R(\widehat{Z}), Q(\widehat{Z})$ and $P(\widehat{Z})$ are all linear functions of $\widehat{Z}$,

$$
\begin{aligned}
& {\left[\mathrm{D}^{2} S(\widehat{Z})-k \mathrm{D}^{2} B(\widehat{Z})-\omega \mathrm{D}^{2} A(\widehat{Z})\right] \widehat{Z}_{\omega}=\boldsymbol{M} \widehat{Z}_{\theta_{3}}} \\
& {\left[\mathrm{D}^{2} S(\widehat{Z})-k \mathrm{D}^{2} B(\widehat{Z})-\omega \mathrm{D}^{2} A(\widehat{Z})\right] \widehat{Z}_{a}=\boldsymbol{M} \mathfrak{g}_{2} .}
\end{aligned}
$$

Now, upon setting $a=\omega=0$ we find that

$$
\left.\boldsymbol{L}(\boldsymbol{p}) \widehat{Z}_{\omega}\right|_{a=\omega=0}=\boldsymbol{M} \mathfrak{g}_{3},\left.\quad \boldsymbol{L}(\boldsymbol{p}) \widehat{Z}_{a}\right|_{a=\omega=0}=\boldsymbol{M g}_{2} .
$$

It is immediate that

$$
V_{21}=\left.\widehat{Z}_{a}\right|_{a=\omega=0}+\operatorname{Ker}(\boldsymbol{L}(\boldsymbol{p})), \quad V_{31}=\left.\widehat{Z}_{\omega}\right|_{a=\omega=0}+\operatorname{Ker}(\boldsymbol{L}(\boldsymbol{p})),
$$

hence, the deformation provides explicit expressions for $V_{21}$ and $V_{31}$.

\section{Secondary criticality and the Benjamin-Feir instability}

We now have enough information to study the leading-order terms in the linear stability problem

$$
L(p) \zeta_{-}=\lambda M \zeta_{-}+\mathrm{i} \alpha \boldsymbol{J} \zeta_{-} .
$$

This equation is solvable if and only if the right-hand side is in $\operatorname{Ker}(\boldsymbol{L}(\boldsymbol{p}))^{\perp}$. This condition provides the dispersion relation between the stability exponent $\lambda$ and the 
modulation wavenumber $\alpha$

$$
\left(\begin{array}{l}
\llbracket \mathfrak{g}_{1}, \lambda \boldsymbol{M} \boldsymbol{\zeta}_{-}+\mathrm{i} \alpha \mathbf{J} \boldsymbol{\zeta}_{-} \rrbracket \\
\llbracket \mathfrak{g}_{2}, \lambda \boldsymbol{M} \boldsymbol{\zeta}_{-}+\mathrm{i} \alpha \boldsymbol{J} \zeta_{-} \rrbracket \\
\llbracket \mathfrak{g}_{3}, \lambda \boldsymbol{M} \zeta_{-}+\mathrm{i} \alpha \boldsymbol{J} \zeta_{-} \rrbracket
\end{array}\right)=0 \quad \text { with } \quad \llbracket \cdot, \cdot \rrbracket:=\oint\langle\cdot, \cdot\rangle \mathrm{d} \theta_{3} .
$$

The function $\zeta_{-}$is an unknown in this equation, but we now have the leading-order terms in the Taylor expansion,

$$
\zeta_{-}=c_{1}\left(\mathfrak{g}_{1}+\mathrm{i} \alpha \widehat{Z}_{h_{0}}\right)+c_{2}\left(\mathfrak{g}_{2}+\lambda \widehat{Z}_{a}+\mathrm{i} \alpha \widehat{Z}_{u_{0}}\right)+c_{3}\left(\mathfrak{g}_{3}+\lambda \widehat{Z}_{\omega}+\mathrm{i} \alpha \widehat{Z}_{k}\right)+O\left(|\lambda|^{2}+|\alpha|^{2}\right) .
$$

Substituting this expansion for $\zeta_{-}$into (6.1) leads to

$$
\begin{aligned}
0 & =\llbracket \mathfrak{g}_{1},(\lambda \boldsymbol{M}+\mathrm{i} \alpha \boldsymbol{J})\left[c_{1}\left(\mathrm{i} \alpha \widehat{Z}_{h_{0}}\right)+c_{2}\left(\lambda \widehat{Z}_{a}+\mathrm{i} \alpha \widehat{Z}_{u_{0}}\right)+c_{3}\left(\lambda \widehat{Z}_{\omega}+\mathrm{i} \alpha \widehat{Z}_{k}\right)\right] \rrbracket+\cdots, \\
0 & =\llbracket \mathfrak{g}_{2},(\lambda \boldsymbol{M}+\mathrm{i} \alpha \boldsymbol{J})\left[c_{1}\left(\mathrm{i} \alpha \widehat{Z}_{h_{0}}\right)+c_{2}\left(\lambda \widehat{Z}_{a}+\mathrm{i} \alpha \widehat{Z}_{u_{0}}\right)+c_{3}\left(\lambda \widehat{Z}_{\omega}+\mathrm{i} \alpha \widehat{Z}_{k}\right)\right] \rrbracket+\cdots, \\
0 & =\llbracket \mathfrak{g}_{3},(\lambda \boldsymbol{M}+\mathrm{i} \alpha \boldsymbol{J})\left[c_{1}\left(\mathrm{i} \alpha \widehat{Z}_{h_{0}}\right)+c_{2}\left(\lambda \widehat{Z}_{a}+\mathrm{i} \alpha \widehat{Z}_{u_{0}}\right)+c_{3}\left(\lambda \widehat{Z}_{\omega}+\mathrm{i} \alpha \widehat{Z}_{k}\right)\right] \rrbracket+\cdots,
\end{aligned}
$$

noting that $\langle U, \boldsymbol{M} V\rangle=\langle U, \boldsymbol{J} V\rangle=0$ whenever $U$ and $V$ are real-valued and $U=V$. In matrix form, this equation becomes

$$
\left[\begin{array}{lll}
N_{11} & N_{12} & N_{13} \\
N_{21} & N_{22} & N_{23} \\
N_{31} & N_{32} & N_{33}
\end{array}\right]\left(\begin{array}{l}
c_{1} \\
c_{2} \\
c_{3}
\end{array}\right)=\left(\begin{array}{l}
0 \\
0 \\
0
\end{array}\right)+O\left((|\lambda|+|\alpha|)^{3}\right) .
$$

Since $\boldsymbol{c}=\left(c_{1}, c_{2}, c_{2}\right)$ is arbitrary, the solvability condition reduces to an algebraic function of $\lambda$ and $\alpha$

$$
\Delta(\lambda, \alpha)=\operatorname{det}\left[\left(\begin{array}{lll}
N_{11} & N_{12} & N_{13} \\
N_{21} & N_{22} & N_{23} \\
N_{31} & N_{32} & N_{33}
\end{array}\right)+O\left((|\lambda|+|\alpha|)^{3}\right)\right]=0 .
$$

In principle, the Taylor series of $\Delta(\lambda, \alpha)$ can be expanded to any order. Convergence of this series is assured in the case of the Boussinesq model, and for weakly nonlinear waves, and it is reasonable to conjecture that the infinite Taylor expansion of $\Delta(\lambda, \alpha)$ converges for almost all values of parameters in the water-wave problem.

The leading-order terms in the Taylor expansion of $\zeta_{-}$provide explicit expressions for the coefficients $N_{i j}$. The first row of the matrix with entries $N_{i j}$ is

$$
\begin{aligned}
& N_{11}=\llbracket \mathfrak{g}_{1},(\lambda \boldsymbol{M}+\mathrm{i} \alpha \boldsymbol{J})\left(\mathrm{i} \alpha \widehat{Z}_{h_{0}}\right) \rrbracket, \\
& N_{12}=\llbracket \mathfrak{g}_{1},(\lambda \boldsymbol{M}+\mathrm{i} \alpha \boldsymbol{J})\left(\lambda \widehat{Z}_{a}+\mathrm{i} \alpha \widehat{Z}_{u_{0}}\right) \rrbracket, \\
& N_{13}=\llbracket \mathfrak{g}_{1},(\lambda \boldsymbol{M}+\mathrm{i} \alpha \boldsymbol{J})\left(\lambda \widehat{Z}_{\omega}+\mathrm{i} \alpha \widehat{Z}_{k}\right) \rrbracket,
\end{aligned}
$$

with similar expressions for the other two rows in the matrix. All these inner products can be simplified. We will give details for the first row,

$$
\begin{aligned}
N_{11} & =\llbracket \mathfrak{g}_{1},(\lambda \boldsymbol{M}+\mathrm{i} \alpha \mathbf{J})\left(\mathrm{i} \alpha \widehat{Z}_{h_{0}}\right) \rrbracket \\
& =\lambda(\mathrm{i} \alpha) \llbracket \mathfrak{g}_{1}, \boldsymbol{M} \widehat{Z}_{h_{0}} \rrbracket+(\mathrm{i} \alpha)^{2} \llbracket \mathfrak{g}_{1}, \boldsymbol{J} \widehat{Z}_{h_{0}} \rrbracket \\
& =-\lambda(\mathrm{i} \alpha) \llbracket \boldsymbol{M g}_{1}, \widehat{Z}_{h_{0}} \rrbracket-(\mathrm{i} \alpha)^{2} \llbracket \boldsymbol{J}_{\mathfrak{g}_{1}}, \widehat{Z}_{h_{0}} \rrbracket \\
& =-(\mathrm{i} \alpha)^{2} \llbracket \nabla R(\widehat{Z}), \widehat{Z}_{h_{0}} \rrbracket \\
& =-(\mathrm{i} \alpha)^{2} \partial R / \partial h_{0},
\end{aligned}
$$


using the fact that $\boldsymbol{M g}_{1}=0$. Similarly,

$$
N_{12}=-\mathrm{i} \alpha \lambda R_{a}-(\mathrm{i} \alpha)^{2} R_{u_{0}}, \quad N_{13}=-\mathrm{i} \alpha \lambda R_{\omega}-(\mathrm{i} \alpha)^{2} R_{k} .
$$

Substituting these expressions into (6.2), the leading-order stability equation (6.2) becomes

$$
\left[\lambda^{2}\left(\begin{array}{ccc}
0 & 0 & 0 \\
0 & 0 & 0 \\
0 & 0 & A_{\omega}
\end{array}\right)+\mathrm{i} \alpha \lambda\left(\begin{array}{ccc}
0 & 1 & A_{h_{0}} \\
1 & 0 & A_{u_{0}} \\
A_{h_{0}} & A_{u_{0}} & 2 A_{k}
\end{array}\right)+(\mathrm{i} \alpha)^{2}\left(\begin{array}{ccc}
R_{h_{0}} & R_{u_{0}} & R_{k} \\
Q_{h_{0}} & Q_{u_{0}} & Q_{k} \\
B_{h_{0}} & B_{u_{0}} & B_{k}
\end{array}\right)\right]\left(\begin{array}{c}
c_{1} \\
c_{2} \\
c_{3}
\end{array}\right)=\left(\begin{array}{c}
0 \\
0 \\
0
\end{array}\right)+\cdots .
$$

For $|\lambda|$ and $|\alpha|$ small, this equation has a non-trivial solution only if the determinant of the coefficient matrix on the left-hand side vanishes. Setting this determinant to zero results in a function of the stability exponent $\lambda$. Let $\lambda=\mathrm{i} \alpha \Omega$. Then the above matrix equation can be written

$$
(\mathrm{i} \alpha)^{2} \mathbf{N}(\Omega) \boldsymbol{c}=\mathbf{0}+O\left((|\lambda|+|\alpha|)^{3}\right),
$$

where $\boldsymbol{N}(\Omega)$ is as defined in $\S 1$. For long-wave instability or Benjamin-Feir instability where $\alpha \neq 0$ but $|\alpha| \ll 1$, the stability analysis therefore reduces to the analysis of $\operatorname{det}(\boldsymbol{N}(\Omega))=0$. The analysis of $\operatorname{det}(\boldsymbol{N}(\Omega))=0$ includes all instabilities for $|\alpha|+|\lambda|$ small, but there is no restriction on the amplitude of the basic state.

The determinant of $\boldsymbol{N}(\Omega)$ can be expanded into the following quartic polynomial

$$
\operatorname{det}[\boldsymbol{N}(\Omega)]=f_{0}+2 f_{1} \Omega+f_{2} \Omega^{2}+2 f_{3} \Omega^{3}+f_{4} \Omega^{4},
$$

with

$$
\begin{aligned}
& f_{0}=\operatorname{det}[\boldsymbol{C}(\boldsymbol{p})], \\
& f_{1}=\left(R_{h} Q_{u}-R_{u}^{2}\right) A_{k}+R_{k} Q_{k}-R_{h} A_{u} Q_{k}-R_{u} B_{k}+A_{h} R_{u} Q_{k}+R_{u} R_{k} A_{u}-A_{h} Q_{u} R_{k}, \\
& f_{2}=\left(R_{h} Q_{u}-R_{u}^{2}\right) A_{\omega}-R_{h} A_{u}^{2}-B_{k}-A_{h}^{2} Q_{u}+2 R_{k} A_{u}+2 A_{h} Q_{k}-4 R_{u} A_{k}+2 A_{h} R_{u} A_{u}, \\
& f_{3}=A_{h} A_{u}-A_{k}-R_{u} A_{\omega}, \\
& f_{4}=-A_{\omega} .
\end{aligned}
$$

For brevity $u$ represents $u_{0}$ and $h$ represents $h_{0}$. The prominent role played by the criticality matrix in this stability quartic is evident.

Given the functionals $R, Q, B$ and $A$ and their derivatives evaluated on the basic state, the stability analysis for long-wave $(\alpha \ll 1)$ instabilities reduces to the analysis of a quartic polynomial. One can deduce general conditions on the coefficients for the existence of at least one root with non-zero imaginary part using the classical theory of quartic equations, but we will not consider this here. In general, for largeamplitude waves, these basic functionals and their derivatives would have to be computed numerically. In $\S 8$, the properties of $\mathbf{N}(\Omega)$ applied to water waves in the limit as the amplitude tends to zero are considered.

\subsection{Linearizing the deformed criticality matrix $\mathbf{N}(\Omega)$}

The 'nonlinear in the parameter' eigenvalue problem associated with $\boldsymbol{N}(\Omega)$ can be linearized. Such matrices are sometimes called $\lambda$-matrices (Lancaster 1966) and they can be linearized as follows. Using Lancaster's linearization when $A_{\omega} \neq 0$,

$$
\operatorname{det}(\boldsymbol{N}(\Omega))=-\frac{1}{A_{\omega}} \operatorname{det}\left[\left(\begin{array}{cccc}
-A_{\omega} & 0 & 0 & 0 \\
0 & R_{h} & R_{u} & R_{k} \\
0 & Q_{h} & Q_{u} & Q_{k} \\
0 & B_{h} & B_{u} & B_{k}
\end{array}\right)+\Omega\left(\begin{array}{cccc}
0 & 0 & 0 & A_{\omega} \\
0 & 0 & 1 & A_{h} \\
0 & 1 & 0 & A_{u} \\
A_{\omega} & A_{h} & A_{u} & 2 A_{k}
\end{array}\right)\right]
$$


The matrix proportional to $\Omega$ on the right-hand side of this expression is invertible. Therefore, when $A_{\omega} \neq 0$, solving $\operatorname{det}(\boldsymbol{N}(\Omega))=0$ is equivalent to finding the eigenvalues of the following $4 \times 4$ non-symmetric matrix

$$
\left(\begin{array}{cccc}
\frac{2}{A_{\omega}}\left(A_{k}-A_{h_{0}} A_{u_{0}}\right) & & \ell^{T} \boldsymbol{C}(\boldsymbol{p}) & \\
A_{u_{0}} & Q_{h_{0}} & Q_{u_{0}} & Q_{k} \\
A_{h_{0}} & R_{h_{0}} & R_{u_{0}} & R_{k} \\
-1 & 0 & 0 & 0
\end{array}\right) \quad \text { where } \quad \ell=\left(\begin{array}{l}
-A_{u_{0}} / A_{\omega} \\
-A_{h_{0}} / A_{\omega} \\
-1 / A_{\omega}
\end{array}\right) .
$$

However, there does not seem to be any advantage to studying the eigenvalues of this matrix rather than analysing $\mathbf{N}(\Omega)$ directly.

\section{Comparison with the Whitham modulation theory}

The Whitham modulation (WM) theory comes closest to capturing the connection between hydraulic properties of the basic state and the BF instability (Whitham 1967, 1974; Hayes 1973). There are, however, two drawbacks to this approach. First, the conservation of total head is not represented, and this forces the WM theory to treat total head as a 'pseudo-frequency', i.e. a temporal parameter. Secondly, the WM theory is developed in a Lagrangian setting, and hence there is insufficient structure to relate bulk quantities to dynamical properties of the equations in a precise way. This problem is circumvented in the WM theory by deriving modulation equations, and then deducing conclusions from the analysis of these modulation equations; but this approach carries with it the need to verify that the modulation equations are indeed valid.

The first problem can be eliminated by introducing a potential for the wave height, but the second problem is intrinsic to the Lagrangian approach. The second problem is partially solved by Hayes (1973) by using a partial Legendre transform from the Lagrangian to the Hamiltonian setting. The multi-symplectic formulation is analogous to a total Legendre transform, which puts space and time on an equal footing.

The WM theory starts with the Lagrangian for water waves and the potential is taken in the form

$$
\phi(x, y, t)=r t+u_{0} x+\widehat{\phi}\left(\theta_{3}\right),
$$

where $\theta_{3}=k x+\omega t$, and the wave height is taken in the form $\eta(x, t)=\widehat{\eta}\left(\theta_{3}\right)$ (compare with Whitham 1974, p. 553, noting that Whitham uses the symbol $-\gamma$ instead of $r$ and $\beta$ instead of $u_{0}$ ). These expressions are then substituted into the Lagrangian averaged over $\theta_{3}$, leading to $\mathscr{L}\left(\omega, k, r, u_{0}\right)$.

The four key parameters in the WM theory are $\omega, r$ which are temporal parameters, and $u_{0}, k$ which are spatial parameters. The parameter $r$ effectively represents the Bernoulli constant (or total head) in the WM theory. Associated with these four parameters are the functionals $\mathscr{L}_{\omega}$ (wave action), $\mathscr{L}_{k}$ (wave action flux), $\mathscr{L}_{r}$ (mass density) and $\mathscr{L}_{u_{0}}$ (mass flux). These functionals are used to derive conservation laws for wave action and mass flux which are then linearized and used to deduce an instability condition. This theory leads to the correct result for instability of water waves even with a mean flow, but the connection with uniform flow hydraulics is missing.

The WM theory can be modified to incorporate conservation of total head. This can be accomplished by introducing a potential for the free surface, and adding an additional pseudofrequency and pseudowavenumber, specifically, let $\eta(x, y, t)=\gamma_{x}$, 
with

$$
\gamma(x, t)=h_{0} x+b t+\widehat{\gamma}\left(\theta_{3}\right)
$$

and replace (7.1) with

$$
\phi(x, t)=u_{0} x+a t+\widehat{\gamma}\left(\theta_{3}\right) .
$$

Then the averaged Lagrangian will be of the form $\mathscr{L}\left(\omega, k, a, u_{0}, b, h_{0}\right)$. The total head is then $\mathscr{L}_{h_{0}}$, and a modulation equation for total head arises naturally when variations of the averaged Lagrangian with respect to $\gamma$ are taken.

With this modified form of the averaged Lagrangian, the WM theory could be rederived, leading to modified modulation equations, which when analysed (linearized about a representation of the basic steady state) should recover the stability theory predicted by the deformed criticality matrix $\mathbf{N}(\Omega)$.

However, there is still the difficulty within the WM theory to relate the conservation laws to the equations directly. This connection is established here by working in the multi-symplectic framework which uses a set of symplectic operators to relate conservation laws directly to symmetry generators and the equations. Indeed, the multi-symplectic framework can be used to derive the stability condition predicted by the WM equations directly from the governing equations (cf. Bridges 1996).

\section{Instability of weakly nonlinear shallow water waves}

In this section, the theory for instability of waves based on $\mathbf{N}(\Omega)$ in (1.1) is applied to weakly nonlinear Stokes waves in finite depth.

Using the expressions for the weakly nonlinear waves in $\S 5$ of Part 1 , and the fact that to leading order the wave action is $A(\widehat{Z})=-D_{\omega}\left|A_{1}\right|^{2}+\cdots$, the entries of the matrix $\mathbf{N}(\Omega)$ in the limit as the amplitude $\left|A_{1}\right| \rightarrow 0$ reduce to

$$
\begin{aligned}
& A_{h_{0}}=\frac{D_{\omega} D_{h_{0}}}{\varrho}, \quad A_{u_{0}}=\frac{D_{\omega} D_{u_{0}}}{\varrho}, \quad A_{k}=\frac{D_{\omega} D_{k}}{\varrho}, \quad A_{\omega}=\frac{D_{\omega} D_{\omega}}{\varrho}, \\
& R_{h_{0}}=g+\frac{D_{h_{0}} D_{h_{0}}}{\varrho}, \quad R_{u_{0}}=u_{0}+\frac{D_{h_{0}} D_{u_{0}}}{\varrho}, \quad R_{k}=\frac{D_{h_{0}} D_{k}}{\varrho}, \\
& Q_{u_{0}}=h_{0}+\frac{D_{u_{0}} D_{u_{0}}}{\varrho}, \quad Q_{k}=\frac{D_{u_{0}} D_{k}}{\varrho}, \quad B_{k}=\frac{D_{k} D_{k}}{\varrho},
\end{aligned}
$$

with the rest of the entries obtained by symmetry. Substituting these expressions into $\mathbf{N}(\Omega)$, we obtain the following form in the limit $\left|A_{1}\right| \rightarrow 0$,

$$
\left(\begin{array}{ccc}
g+\frac{D_{h} D_{h}}{\varrho} & u_{0}+\frac{D_{h} D_{u}}{\varrho} & \frac{D_{h} D_{k}}{\varrho} \\
u_{0}+\frac{D_{u} D_{h}}{\varrho} & h_{0}+\frac{D_{u} D_{u}}{\varrho} & \frac{D_{u} D_{k}}{\varrho} \\
\frac{D_{k} D_{h}}{\varrho} & \frac{D_{k} D_{u}}{\varrho} & \frac{D_{k} D_{k}}{\varrho}
\end{array}\right)+\left(\begin{array}{ccc}
0 & 1 & \frac{D_{\omega} D_{h}}{\varrho} \\
1 & 0 & \frac{D_{\omega} D_{u}}{\varrho} \\
\frac{D_{\omega} D_{h}}{\varrho} & \frac{D_{\omega} D_{u}}{\varrho} & 2 \frac{D_{\omega} D_{k}}{\varrho}
\end{array}\right) \Omega+\frac{D_{\omega}^{2}}{\varrho} \boldsymbol{e}_{3} \boldsymbol{e}_{3}^{T} \Omega^{2},
$$

again suppressing subscripts in subscripts, with determinant

$$
\operatorname{det}(\mathbf{N}(\Omega))=-\frac{D_{\omega}^{2}}{\varrho}\left(\Omega+\frac{D_{k}}{D_{\omega}}\right)^{2}\left(\Omega+u_{0}+\sqrt{g h_{0}}\right)\left(\Omega+u_{0}-\sqrt{g h_{0}}\right) .
$$



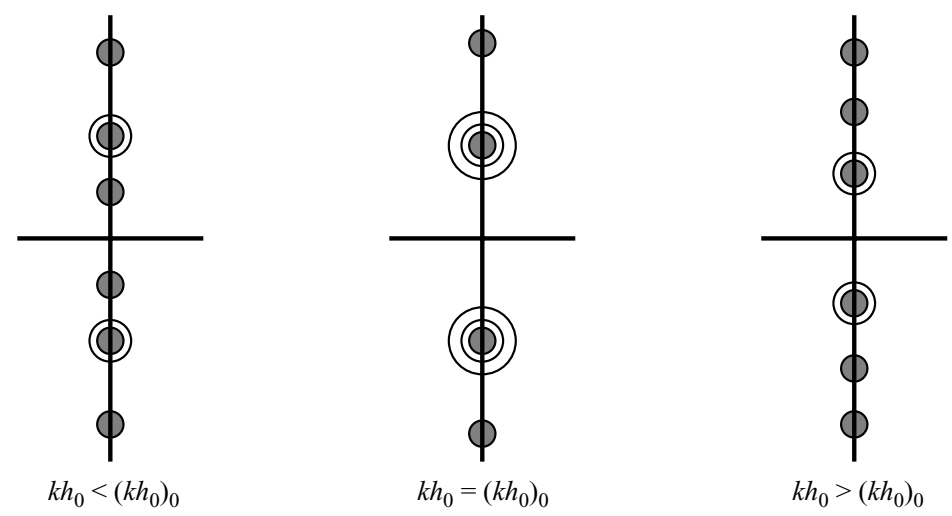

FIGURE 1. Schematic of the position of the temporal eigenvalues in the complex $\lambda$-plane as $k h_{0}$ is varied. Here, $\lambda=\mathrm{i} \Omega$ and $\Omega$ is a root of $\operatorname{det}(\boldsymbol{N}( \pm \Omega))=0$ with $\left|A_{1}\right|=0$. The critical value $\left(k h_{0}\right)_{0}$ is approximately 2 .

Hence $-D_{k} / D_{\omega}$ is a root of multiplicity two, and there are two simple roots. The root $-D_{k} / D_{\omega}$ plays a role similar to a group velocity, but it does not equal the group velocity for water waves in shallow water because $\omega$ is a modulation parameter here, specifically $-D_{k} / D_{\omega}=c_{g}-u_{0}$.

Going back to (3.4) and (3.5), we see that in addition to $\operatorname{det}(\boldsymbol{N}(\Omega))=0$ we have to add the four roots of $\operatorname{det}(\boldsymbol{N}(-\Omega))=0$. This gives eight roots and they are plotted in figure 1 .

The only curiosity in this figure is that there is a critical value of $k h_{0} \approx 2$ at which there is a triple eigenvalue. However, this triple eigenvalue is harmless and does not lead to instability, as the eigenvector of the simple eigenvalue which collides with the double eigenvalue is linearly independent through the collision. This can be seen by noting that the roots of $\operatorname{det}(\boldsymbol{N}(\Omega))=0$ are

$$
\left.-\frac{D_{k}}{D_{\omega}}<0, \quad-u_{0}-\sqrt{g h_{0}}<0, \quad-u_{0}+\sqrt{g h_{0}}>0 \quad \text { (assuming } u_{0}^{2}<g h_{0}\right),
$$

and the roots of $\operatorname{det}(\boldsymbol{N}(-\Omega))=0$ are the negative of these. Therefore, the triple collision involves a double root from one determinant and a simple root from the other, and appeal to (3.5) shows that there will be two independent eigenvectors at the triple collision.

The algebraic properties of $\mathbf{N}(\Omega)$ in the limit $\left|A_{1}\right| \rightarrow 0$ are as follows. Let $\Omega_{0}=-D_{k} / D_{\omega}$ and let $\boldsymbol{N}_{0}=\boldsymbol{N}\left(\Omega_{0}\right)$; then

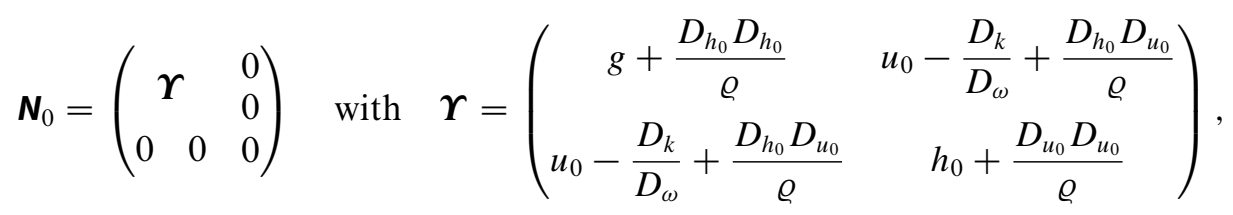

with

$$
\operatorname{det}(\boldsymbol{\Upsilon})=\left(g h_{0}-c_{g}^{2}\right) \frac{\widehat{\varrho}}{\varrho} \quad \text { where } \widehat{\varrho}=\varrho+\left\langle\left(\begin{array}{c}
D_{h_{0}} \\
D_{u_{0}}
\end{array}\right),\left(\begin{array}{cc}
g & c_{g} \\
c_{g} & h_{0}
\end{array}\right)^{-1}\left(\begin{array}{c}
D_{h_{0}} \\
D_{u_{0}}
\end{array}\right)\right\rangle .
$$

The symbol $c_{g}$ here should be interpreted as representing $c_{g}=u_{0}-D_{k} / D_{\omega}$. 
When $\left|A_{1}\right|$ is perturbed away from zero, the only way that instability can arise, starting with the eigenvalue configuration in figure 1 , is for the double eigenvalues at $\pm \mathrm{i} D_{k} / D_{\omega}$ to split and develop a non-zero real part. With an elementary perturbation argument, it can be shown that when $0<\left|A_{1}\right| \ll 1$ there is a $\operatorname{root}$ of $\operatorname{det}(\boldsymbol{N}(\Omega))=0$ of the form

$$
\Omega=\Omega_{0} \pm \frac{1}{D_{\omega}^{2}} \sqrt{-\widehat{\varrho} \widehat{\delta}}\left|A_{1}\right|+O\left(\left|A_{1}\right|^{2}\right),
$$

where $\widehat{\delta}$ is the determinant introduced in equation (4.6), and $\widehat{\varrho}$ is defined in (8.3). It follows that there is a root of $\operatorname{det}(\boldsymbol{N}(\Omega))=0$ with non-zero imaginary part (hence instability) when $\widehat{\delta} \widehat{\varrho}>0$.

The form of the root $\Omega$ in (8.4) is confirmed by using an elementary perturbation analysis, with perturbation parameter $\left|A_{1}\right|$, of the nonlinear (in the parameter) eigenvalue problem

$$
\mathbf{N}(\Omega) \zeta=0, \quad \zeta \in \mathbb{C}^{3}
$$

by taking $\Omega=\Omega_{0}+\Omega_{1}\left|A_{1}\right|+\Omega_{2}\left|A_{1}\right|^{2}+\cdots$, and

$$
\zeta=\zeta_{0}+\left|A_{1}\right| \zeta_{1}+\left|A_{1}\right|^{2} \zeta_{2}+\cdots, \quad \mathbf{N}(\Omega)=\mathbf{N}_{0}+\left|A_{1}\right| \mathbf{N}_{1}+\left|A_{1}\right|^{2} \mathbf{N}_{2}+\cdots,
$$

with $\boldsymbol{N}_{0}$ as defined in (8.2). A straightforward calculation then leads to $D_{\omega}^{4} \Omega_{1}^{2}=-\widehat{\varrho} \widehat{\delta}$.

In Hasimoto \& Ono (1972), it is shown that a model for the modulation of Stokes waves in finite depth is the NLS equation

$$
\mathrm{i} A_{\tau}+\mu A_{\xi \xi}+\widetilde{v}|A|^{2} A=0 .
$$

The coefficients and properties of this equation are discussed in $\S 10$. From the results of Hasimoto \& Ono (1972) it is easy to check that $\mu / \widehat{\delta}$ is a positive constant, and $\widetilde{v} / \widehat{\varrho}$ is a positive constant. Hence the instability condition $\widehat{\delta} \widehat{\varrho}>0$ agrees with the instability condition $\mu \widetilde{v}>0$ predicted by the NLS model (Hasimoto \& Ono 1972), which in turn agrees with the result first found by Benjamin (1967), and confirmed using the Whitham modulation theory (Whitham 1967), and rigorously demonstrated in Bridges \& Mielke (1995).

Indeed, the instability of weakly nonlinear Stokes waves based on the deformed criticality matrix could be rigorously justified using a modification of the centremanifold theory in Bridges \& Mielke (1995). Technically, the theory would go through, with the only requirement being that the conservation of total head has to be incorporated which would increase the dimension of the centre-manifold from four to six.

The critical coefficient $\widehat{\varrho}$ is the one that changes sign when $k h_{0}=\left(k h_{0}\right)^{\text {crit }} \approx 1.36$. For $k h_{0}<\left(k h_{0}\right)^{c r i t}$, the parameter $\widehat{\varrho}$ is positive. Since $\varrho$ is negative for all $k h_{0}$, it is the second term in $\widehat{\varrho}$ in $(8.3)$,

$$
\left\langle\left(\begin{array}{c}
D_{h_{0}} \\
D_{u_{0}}
\end{array}\right),\left(\begin{array}{cc}
g & c_{g} \\
c_{g} & h_{0}
\end{array}\right)^{-1}\left(\begin{array}{c}
D_{h_{0}} \\
D_{u_{0}}
\end{array}\right)\right\rangle,
$$

which is due to the contribution from the criticality matrix with $u_{0}$ replaced by $c_{g}$, that stabilizes the Benjamin-Feir instability. In other words, it is the uniform flow i.e. mean flow - which stabilizes the perturbation. A schematic of the position of the unstable eigenvalues for $k h_{0}>\left(k h_{0}\right)^{c r i t}$ is shown in figure 2. 

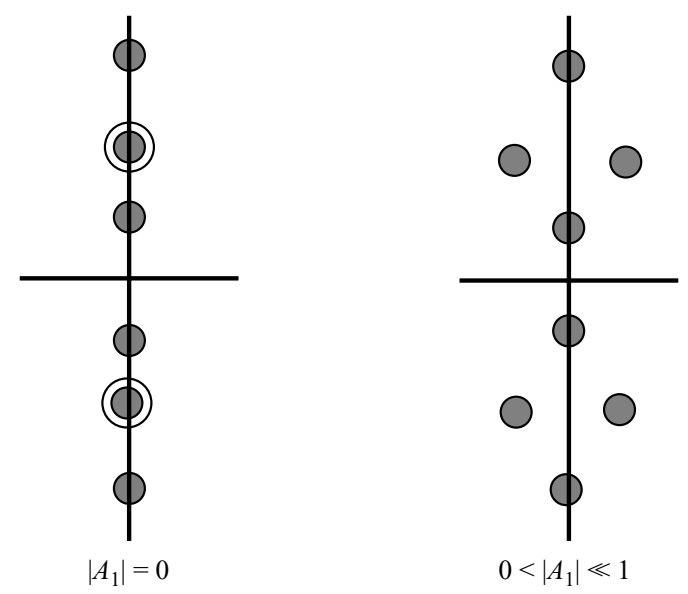

FIGURE 2. Schematic of the position of the temporal eigenvalues in the complex $\lambda$-plane when $\left|A_{1}\right|$ is perturbed away from zero, when $k h_{0}$ is between $\left(k h_{0}\right)^{c r i t} \approx 1.36$ and $\left(k h_{0}\right)_{0} \approx 2$.

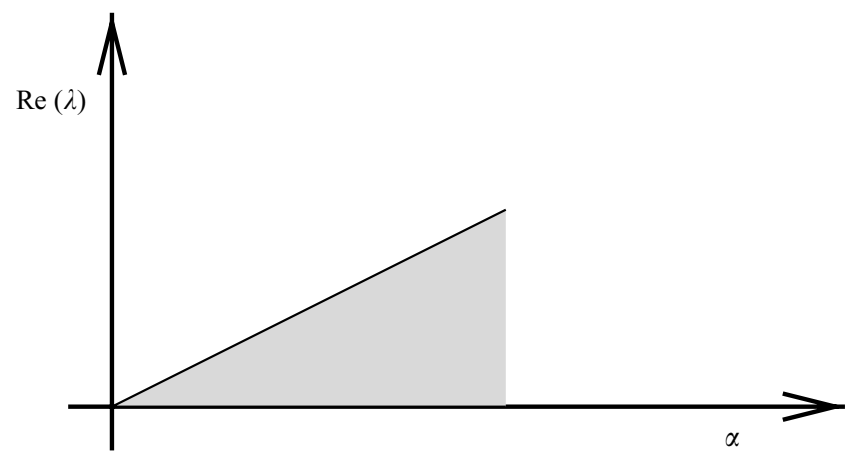

FIGURE 3. The band of unstable stability exponents $\operatorname{Re}(\lambda)$ as a function of the modulation wavenumber $\alpha$.

\section{The bandwidth of unstable wavenumbers}

A root of the determinant of $\mathbf{N}(\Omega)$ with non-zero imaginary part indicates the existence of a band of unstable eigenvalues as a function of $\alpha$ as shown in figure 3 . Therefore, this condition is a sufficient, but not necessary condition for instability. If all roots of the determinant of $\mathbf{N}(\Omega)$ are real, then there does not exist an unstable eigenvalue for $\alpha$ near zero, but there could exist unstable eigenvalues at larger values of $\alpha$. When $k h_{0}<\left(k h_{0}\right)^{\text {crit }}$, instabilities at larger values of $\alpha$ are known to exist from numerical calculations (see figure $2 d$ of McLean 1982 and figures 1 and 2 in Francius \& Kharif 2003).

To determine the bandwidth of the unstable wavenumbers, or to determine whether there are instabilities with larger $|\alpha|$, one can in principle expand the Taylor series of $\Delta(\lambda, \alpha)$ to any order. This expansion is straightforward in the weakly nonlinear case, and leads to the well-known cutoff wavenumber first discovered by Benjamin (1967). The Whitham modulation theory can also be carried to the next order to determine the cutoff value. The extension of the WM theory to the next order is discussed in (Hayes 1973, p. 218) (called 'diffraction effect' there). In the weakly nonlinear case, the details of this expansion were carried out in Yuen \& Lake (1975) (see Fornberg \& Whitham 1978, §8), leading to a variational derivation of the NLS equation. 
However, at finite amplitude, the value of the cutoff wavenumber is known only in some cases. Numerical results of McLean (1982) and Francius \& Kharif (2003) show values of the cutoff wavenumber for some special parameter values, but a systematic study of the effect of mean flow on bandwidth is largely an open question.

In this paper, we have considered longitudinal instabilities; that is, instabilities that propagate in the same direction as the basic state. However, shallow-water waves are also susceptible to oblique instabilities (cf. Hayes 1973; Bryant 1978; McLean 1982).

\section{Unsteady dark solitary waves}

When the critical coefficient $\widetilde{v}$ in the NLS equation (8.5) changes sign in going from $k h_{0}>k h_{0}^{\text {crit }} \approx 1.363$ to $k h_{0}<k h_{0}^{\text {crit }}$ the type changes from focusing NLS to de-focusing NLS. It is well-known that the de-focusing NLS has DSW solutions, and so in the context of water waves, there are weakly nonlinear unsteady DSWs for $k h_{0}<k h_{0}^{\text {crit }}$. The purpose of the present discussion is to contrast these solitary waves with the steady DSWs discovered in Part 1.

Consider the coupled NLS model for modulation of Stokes waves in finite depth derived by Hasimoto \& Ono (1972)

$$
\begin{aligned}
\frac{\partial^{2} \varphi}{\partial T^{2}}-g h_{0} \frac{\partial^{2} \varphi}{\partial X} & =\frac{g}{k_{0} \sigma}\left[2 \omega_{0} k_{0}+\left(1-\sigma^{2}\right) k_{0}^{2} c_{g}\right]\left(|A|^{2}\right)_{\xi}, \\
\mathrm{i} \frac{\partial A}{\partial \tau}+\mu \frac{\partial^{2} A}{\partial \xi^{2}}+\nu|A|^{2} A & =\left(k_{0} \frac{\partial \varphi}{\partial X}-\left(1-\sigma^{2}\right) \frac{k_{0}^{2}}{2 \omega_{0}} \frac{\partial \varphi}{\partial T}\right) A,
\end{aligned}
$$

where $\sigma=\tanh \left(k_{0} h_{0}\right), c_{g}$ is the group velocity, $\mu=\frac{1}{2} \omega^{\prime \prime}\left(k_{0}\right), T=\varepsilon t, X=\varepsilon x, \tau=\varepsilon^{2} t$ and $\xi=X-c_{g} T$, and

$$
v=\frac{1}{2} \frac{\omega_{0} k_{0}^{2}}{\sigma^{2}}\left[\left(1-\sigma^{2}\right)^{2}-\frac{1}{2 \sigma^{2}}\left(9-10 \sigma^{2}+9 \sigma^{4}\right)\right] .
$$

The function $A(\xi, \tau)$ represents modulation of the Stokes wave, and $\varphi$ represents modulation of the meanflow. (The symbol $A$ here is not to be confused with wave action. Since wave action is not discussed in this section there is no cause for confusion.) Hasimoto \& Ono eliminate $\varphi$ by assuming it to be a function of $\xi$ only, then

$$
\left(g h_{0}-c_{g}^{2}\right) \varphi_{\xi}=C_{0}+C_{1}|A|^{2},
$$

where $C_{0}$ is a constant of integration, and $C_{1}$ can be determined from (10.1). The value of $C_{0}$ is related to the choice of mass flux (it can in principle also be a function of time), but it is customary to set $C_{0}=0$ without explanation (see equation (4.4) of Hasimoto \& Ono 1972). Sedletsky (2005) has reappraised the theory of Hasimoto \& Ono without this assumption and without the assumption that $\varphi$ is a function of $\xi$, and makes several interesting observations. However, the role of $C_{0}$ is not important for the present argument and so is set to zero. (See Bridges (2005) for a discussion of the implications of non-zero $C_{0}$.)

Substituting (10.2) into $(10.1 b)$ results in

$$
\mathrm{i} A_{\tau}+\mu A_{\xi \xi}+\widetilde{v}|A|^{2} A=0 .
$$


There are no steady DSW solutions of this equation, but when $\widetilde{v}>0$ there is a family of unsteady DSWs, with explicit expression

$$
A(\xi, \tau)=\mathrm{e}^{\mathrm{i}(\tilde{k} \xi-\tilde{\omega} \tau)}\left(\frac{2 \mu^{2}}{|\mu \widetilde{v}|}\right)^{1 / 2}(\tilde{k}+\mathrm{i} \chi \tanh (\chi \xi)), \quad \chi^{2}=\frac{1}{2 \mu^{2}}\left(\mu \tilde{\omega}-3 \mu^{2} \tilde{k}^{2}\right) .
$$

with the requirement $\mu \tilde{\omega}>3 \mu^{2} \tilde{k}^{2}$.

Although these DSWs are mathematically similar to the steady DSWs in Part 1, they are intrinsically unsteady. The leading-order expression for the waveheight for steady DSWs is

$$
\eta(x, t)=\varepsilon \operatorname{Re}\left(A(\xi, \tau) \exp \left(\mathrm{i}\left(k_{0} x-\omega_{0} t\right)\right)\right) .
$$

There are two frame speeds: the phase speed of the Stokes wave, and the frame speed of the DSW which is the group velocity, and further unsteadiness due to the $\mathrm{e}^{-\mathrm{i} \tilde{\omega} \tau}$ term. In fact, the modulation equation of Hasimoto \& Ono, and related modulation equations for Stokes waves in finite depth are intrinsically unsteady, and therefore do not capture the steady DSWs that are synchronized with the Stokes wave (cf. Bridges 2005).

These unsteady DSWs arise because of the change in BF instability of the Stokes wave. According to a weakly nonlinear NLS analysis, they exist for $k h_{0}$ less than about 1.36. However, it has never been confirmed analytically or numerically that these unsteady DSWs are solutions of the full water-wave problem, and there are no known experimental or field observations of unsteady DSWs.

The steady DSWs of Part 1 are steady relative to an absolute frame of reference, and can be found by direct analysis of the water-wave problem, or with model equations such as the Boussinesq equation. In the weakly nonlinear limit, they exist for $k h_{0}$ less than about 0.85 . The steady DSWs have a simple physical interpretation. They are Stokes waves with a region of localized modulation. In an experiment or field observation, they would most probably look like Stokes waves predominantly, but would have a small region of modulation, as shown in figure 1 of Part 1 .

\section{Summary and conclusions}

Criticality is a central organizing concept in open-channel hydraulics. The purpose of the present work was two-fold: to generalize criticality to open-channel flows which are spatially varying in the streamwise direction, and to identify the implications of criticality for the physical system.

Heretofore the only generalizations of criticality for spatially varying flows have resulted in approximate methods. The generalization proposed here is exact for spatially periodic variation, with the principal example being wave formation on uniform flows. The central outcome in the theory is the criticality matrix introduced in Part 1.

The justification of the criticality matrix brings together ideas from hydraulics, dynamical systems, conservation laws, group theory, linear algebra, constrained optimization and Hamiltonian dynamics. However, the resulting theory of steady criticality is very close to the physical aspects of the flow and is easy to apply, being based on four physically important functionals: total head, flow force, mass flux and wave action flux, and their dependence on three parameters: mean depth, mean velocity, and wavelength.

One of the principal motivations for generalizing criticality was to determine its consequences. For the steady problem, we found that criticality predicts the 
movement of eigenvalues in the linearization about a basic spatially varying flow. Using dynamical systems theory, we were able to deduce behaviour in the nonlinear problem near the basic state as well, namely, the bifurcation of homoclinic orbits, which represent solitary waves with oscillatory tails - a form of steady dark solitary wave (DSW) - in the flow field.

Applying this theory to water waves showed that indeed secondary criticality is very relevant to finite-depth water waves, particularly at intermediate depths. We found that secondary criticality occurs along branches of shallow-water Stokes waves on a uniform flow - at low amplitude and for a wide range of parameters. The theory shows that steady DSWs play a much greater role in shallow-water wave theory than previously thought. The theory is consistent with previous numerical work of Vandenbroeck (1983) and Zufiria (1987), and is consistent with the appearance of DSWs in the NLS equation for intermediate depths, although the DSWs in the NLS equation are unsteady. Our theory ties together previous work and provides a unifying explanation for both the appearance of spatial Floquet multipliers passing through +1 , and the appearance of steady DSWs. Both phenomena are a consequence of secondary criticality.

As far as we are aware, the concept of criticality for unsteady flows has never been considered heretofore. The framework for criticality proposed in Part 1, based on the theory of relative equilibria, has a straightforward generalization to unsteady flows, and an example of this theory was presented here in Part 2. The consequence of the theory for unsteady criticality is a new perspective on the BF instability in shallow water. Although it is known that mean flow acts as a stabilizing influence on the BF instability, the precise connection between the hydraulic theory of uniform flows and the BF instability presented in Part 2 is new.

Other outcomes of the theory in the work which may have independent interest are $(a)$ the characterization of the Bernoulli equation as a conservation law; $(b)$ the identification of a symmetry which can be associated with the Bernoulli equation; (c) extension of the $S Q R$-theory of Benjamin-Lighthill to the $S Q R+B$ theory; (d) the general connection between uniform flows, and other basic states in openchannel flow, with symmetry and relative equilibria; $(e)$ the precise parameter structure of water waves in shallow water, identifying exactly three characterizing pairs of parameters in the steady case; $(f)$ a framework where the effect of mean flow on instability can be systematically studied; $(g)$ simple formulae for the qualitative properties of bifurcating classical solitary waves and steady dark solitary waves.

There are a number of further directions that are suggested by the theory in this paper; one is to apply the theory to stratified fluids, where issues of criticality are paramount, and a classical view of criticality as a one-parameter problem fails. A good starting point in this direction is two-layer flows with a free surface, where the methodology is very similar to this paper. For example, uniform flows will have five functionals: total head and mass flux in each layer, and flow force. Uniform two-layer flows coupled with periodic waves will additionally have a wave-action flux, and so six functionals, with five Lagrange multipliers (mean depth in each layer, mean velocity in each layer and wavenumber). We can see from looking at uniform flows, that the potential bifurcations and creation of solitary waves will be much richer. For example, for uniform flows, the $(S, Q)$ curve in figure 4 of Part 1 generalizes to a surface in $\left(S, Q_{1}, Q_{2}\right)$-space, with cusp lines rather than cusp points. Therefore, for fixed total head in each layer, there are curves of criticality rather than points of criticality. The potentialities and consequences of secondary criticality, bifurcation of classical solitary waves and steady dark solitary wave, and instability of interfacial waves, are manifold. 


\section{Appendix. Expressing wave action in terms of mass flux for water waves}

In the stability matrix $\boldsymbol{N}(\Omega)$, the only functional which does not appear in the strictly steady problem is the wave action density $A$. However, for water waves, this functional can be expressed in terms of $Q$, and thereby stating all aspects of the stability in terms of hydraulic quantities known from the steady problem.

For a periodic wave of the form introduced in $\S 5$ of Part 1 ,

$$
Q(\widehat{Z})=\oint \int_{0}^{\widehat{\eta}} \widehat{u} \mathrm{~d} z \mathrm{~d} \theta_{3}, \quad A(\widehat{Z})=-\oint \widehat{\Phi} \frac{\partial \widehat{\eta}}{\partial \theta_{3}} \mathrm{~d} \theta_{3}=\oint \int_{0}^{\widehat{\eta}} \frac{\partial \widehat{\phi}}{\partial \theta_{3}} \mathrm{~d} z \mathrm{~d} \theta_{3},
$$

noting that $\widehat{\phi}$ is a periodic function of $\theta_{3}$. However, $\widehat{u}=u_{0}+k \widehat{\phi}_{\theta_{3}}$, and so

$$
\begin{aligned}
Q(\widehat{Z}) & =\oint \int_{0}^{\widehat{\eta}}\left(u_{0}+k \frac{\partial \widehat{\phi}}{\partial \theta_{3}}\right) \mathrm{d} z \mathrm{~d} \theta_{3} \\
& =u_{0} \oint \widehat{\eta} \mathrm{d} \theta_{3}+k \oint \int_{0}^{\widehat{\eta}} \frac{\partial \widehat{\phi}}{\partial \theta_{3}} \mathrm{~d} z \mathrm{~d} \theta_{3} \\
& =u_{0} h_{0}+k A(\widehat{Z}),
\end{aligned}
$$

providing an explicit relation for expressing derivatives of $A$ in terms of derivatives of $Q$ :

$$
A_{h_{0}}=\frac{1}{k}\left(Q_{h_{0}}-u_{0}\right), \quad A_{u_{0}}=\frac{1}{k}\left(Q_{u_{0}}-h_{0}\right), \quad A_{k}=\frac{1}{k}\left(Q_{k}-A\right), \quad A_{\omega}=\frac{1}{k} Q_{\omega} .
$$

\section{REFERENCES}

Benjamin, T. B. 1967 Instability of periodic wavetrains in nonlinear dispersive systems. Proc. $R$. Soc. Lond. A 299, 59-75.

Benjamin, T. B. 1984 Impulse, flow force and variational principles. IMA J. Appl. Maths 32, 3-68.

BRIDGES, T. J. 1996 Periodic patterns, linear instability, symplectic structure and mean-flow dynamics for three-dimensional surface waves. Phil. Trans. R. Soc. Lond. A 354, 533-74.

Bridges, T. J. 1997 Multi-symplectic structures and wave propagation. Math. Proc. Camb. Phil. Soc. 121, 147-190.

BRIDGES, T. J. 2004 On the susceptibility of bright NLS solitons to long-wave transverse instability. Proc. R. Soc. Lond. A 460, 2605-2515.

BRIDGES, T. J. 2005 Steady dark solitary waves emerging from wave-generated meanflow: the role of modulation equations. Chaos 15, 037113.

Bridges, T. J. \& Donaldson, N. M. 2006 Secondary criticality of water waves. Part 1. Definition, bifurcation and solitary waves. J. Fluid Mech. 565, 381-417.

Bridges, T. J. \& Mielke, A. 1995 A proof of the Benjamin-Feir instability. Arch. Rat. Mech. Anal. 133, 145-198.

Bryant, P. J. 1978 Oblique instabilities of periodic waves in shallow water. J. Fluid Mech. 86, 783-792.

Dingemans, M. W. 1997 Water Wave Propagation over Uneven Bottoms. Part 2 - Non-linear Wave Propagation. World Scientific.

Fornberg, B. \& Whitham, G. B. 1978 A numerical and theoretical study of certain nonlinear wave phenomena. Phil. Trans. R. Soc. Lond. A 289, 373-404.

Francius, M. \& Kharif, C. 2003 On the disappearance of the lowest-order instability for steep gravity waves in finite depth. Phys. Fluids 15, 2445-2448.

Hasimoto, H. \& Ono, H. 1972 Nonlinear modulations of gravity waves. J. Phys. Soc. Japan 33, 805-811.

HaYes, W. D. 1973 Group velocity and nonlinear dispersive wave propagation. Proc. R. Soc. Lond. A 332, 199-221.

Lancaster, P. 1966 Lambda Matrices and Vibrating Systems. Pergamon. 
MCLean, J. W. 1982 Instabilities of finite-amplitude gravity waves on water of finite depth. J. Fluid Mech. 114, 331-341.

Magnus, J. R. \& Neudecker, H. 1988 Matrix Differential Calculus. John Wiley.

Peregrine, D. H. 1983 Water waves, nonlinear Schrödinger equations and their solutions. J. Austral. Math. Soc. B 25, 16-43.

SedLETSKy, YU. V. 2005 The modulational instability of Stokes waves on the surface of finite-depth fluid. Phys. Lett. A 343, 293-299.

Vandenbroeck, J.-M. 1983 Some new gravity waves in water of finite depth. Phys Fluids 26, 2385-2387.

Whitham, G. B. 1967 Nonlinear dispersion of water-waves. J. Fluid Mech. 27, 399-412.

Whitham, G. B. 1974 Linear and Nonlinear Waves. Wiley-Interscience.

Yuen, H. C. \& LaKe, B. M. 1975 Nonlinear deep water waves: theory and experiment. Phys. Fluids 18, 956-960.

ZuFIRIA, J. A. 1987 Weakly nonlinear non-symmetric gravity waves on water of finite depth. J. Fluid Mech. 180, 371-385. 